\title{
Tailoring Dual Antiplatelet Therapy after Coronary Stenting: The PRECISE-DAPT Score versus the DAPT Score
}

\section{Cheuk-Kit Wong}

Department of Medicine and Therapeutics, The Chinese University of Hong Kong, Hong Kong

*Corresponding author: Cheuk-Kit Wong, Department of Medicine and Therapeutics, Faculty of Medicine, Prince of Wales Hospital Shatin, The Chinese University of Hong Kong, Hong Kong, Tel: 852-35053139; E-mail: cheuk-kit.wong@cuhk.edu.hk

Received date: August 01, 2017; Accepted date: August 03, 2017; Published date: August 08, 2017

Copyright: (c) 2017 Wong CK. This is an open-access article distributed under the terms of the Creative Commons Attribution License, which permits unrestricted use, distribution, and reproduction in any medium, provided the original author and source are credited.

\section{Editorial}

Both aspirin mono-therapy and dual aspirin $-\mathrm{P}_{2} \mathrm{Y}_{12}$ receptor antagonist therapy (DAPT) are commonly used for coronary disease. The use of DAPT post-coronary stenting is standard. The benefit-torisk assessment for longer versus shorter duration of DAPT depends on the coronary ischemic risks, the nature of antiplatelet medications and the general bleeding risks. Previous editorials in the journal have addressed in part the former 2 issues [1-3] and the current editorial will focus on the last and integrate them in clinical application.

\section{The PRECISE-DAPT score: predicting out-of hospital bleeding post-coronary stenting}

This score was derived using patient-level data among 14963 clinical trial patients treated with DAPT post-coronary stenting (patients requiring oral anticoagulation excluded) from 8 multicentre randomised trials [4]. All relevant TIMI major or minor bleeding events were independently adjudicated. Using Cox proportional hazards regression, multivariable predictors for out-of-hospital bleeding occurring $\geq 7$ days after the initial stenting procedure were obtained on which the numerical PRECISE-DAPT bleeding risk score was developed. PRECISE-DAPT consists of 4 continuous parameters scored on a linear or near linear scale (listed from zero to maximum value in score calculation: age ranging from 50 to 90 years, creatinine clearance ranging from 100 to $0 \mathrm{ml} / \mathrm{min}$, haemoglobin ranging from 12 to $10 \mathrm{~g} / \mathrm{dL}$, white-blood-cell count ranging from 5 to $20 \times 10^{3}$ cells per $\mu \mathrm{l})$, and the dichotomous parameter previous spontaneous bleeding.

The predictive performance of the PRECISE-DAPT score was examined in patients treated with percutaneous coronary intervention (PCI) from the PLATO trial involving 8595 patients and from the BernPCI registry involving 6172 patients. In the derivation cohort PRECISE-DAPT had a C-index of 0.73 (95\% CI 0.61-0.85) predicting out-of-hospital TIMI major or minor bleeding. The corresponding Cindex was $0.70(0.65-0.74)$ in the PLATO trial validation cohort and $0.66(0.61-0.71)$ in the BernPCI registry validation cohort. The score also performed well in predicting TIMI major bleeding, albeit with a lower C-index in all the examined cohorts.

DAPT duration was randomly allocated in 5 of the 8 studies included in the derivation cohort involving 10,081 of the 14,963 patients. A longer DAPT duration (i.e., 12-24 months versus 3-6 months) was found to significantly increase bleeding in patients with higher PRECISE-DAPT score $(\geq 25)$, but not in those with lower $(<25)$ score; (Interaction P value 0.007), an expected finding as PRECISEDAPT score reflected bleeding risks. Of interest, the anti-ischemic benefit of a longer DAPT duration was observed only in the latter (lower bleeding risks) patients. Patients with a PRECISE-DAPT score $\geq 25$ represented the upper quarter of the cohort.

To provide an idea in PRECISE-DAPT score calculation, a history of previous spontaneous bleeding alone will give 25 points; an age of 75 and having creatinine clearance of $30 \mathrm{ml} / \mathrm{min}$ and haemoglobin level of $11.1 \mathrm{~g} / \mathrm{dl}$ will give 12,18 and 6 points respectively with a total of 36 . The investigators also evaluated a simplified 4 factor score "PRECISEDAPT alternative" which removed the white-blood-cell count factor and found a similar C-index for predicting bleeding in the PLATO validation cohort and a slight reduced C-index in the BERN-PCI validation cohort.

C-index is a statistical method to evaluate how good the parameter/ algorithm predicts outcome. A C-index of 1 means correct prediction all of the times while a C-index of 0.5 means the prediction parameter/ algorithm is no better than any random guess. A C-index of around 0.70 (as obtained by the PRECISE-DAPT score predicting bleeding) means modest prediction ability. The PRECISE-DAPT score is a step forward pulling together "cumulative" effects of the major bleeding factors but there is no consideration of the underlying ischemia risks.

\section{The DAPT score: predicting net effect of DAPT considering both ischemia and bleeding factors for patients who have uneventfully tolerated a year of DAPT post- stenting}

The DAPT trial report [5], described a randomized cohort of 9961 patients who had already tolerated a year of aspirin plus thienopyridine (2/3 clopidogrel, 1/3 prasugrel) post- Drug-Eluting Stent (DES) implantation without any significant ischemic or bleeding events. Randomization involved either continuing the same DAPT regime or taking aspirin plus placebo in lieu of thienopyridine for another 18 months. Patients taking oral anticoagulants were excluded. Among these 9,961 patients $26 \%$ presented initially with acute myocardial infarction and $51 \%$ had $\geq 1$ clinical or angiographic risk factor for stent thrombosis. The types of DES included the currently unavailable/withdrawn paclitaxel eluting stent which is now known to cause poor coronary healing with very late stent thrombosis [1-3]. Over the DAPT Study period (12 to 30 months post-stenting), continued thienopyridine (versus placebo) significantly reduced the composite endpoint of death, myocardial infarction, or stroke $(4.3 \% \mathrm{vs}$. $5.9 \%)$ but increased the rate of GUSTO moderate or severe bleeding (2.5\% vs. $1.6 \%)$.

To dissect the competing ischemic and bleeding risks, the investigators developed the DAPT score based on the "parent" DAPT Study cohort of 11,648 patients (adding the patients who received bare metal stent instead of DES) in the period from 2009 August to 2014 May. External validation was performed using 8136 patients 
randomized in the PROTECT trial in the period from 2007 June to 2014 July [6].

The DAPT score assigns 1 point each for myocardial infarction at presentation, prior myocardial infarction or PCI, diabetes, stent diameter $<3 \mathrm{~mm}$, smoking, and paclitaxel-eluting stent; 2 points each for history of congestive heart failure/low ejection fraction and vein graft intervention; - 1 point for age 65 to 75 years; and -2 points for age $\geq 75$ years. Because peripheral arterial disease, renal insufficiency, and hypertension were found to predict both ischemic and bleeding events, they were not scored. The positive DAPT score factors includes clinical (myocardial infarction, heart failure, diabetes and smoking) and angiographic/ procedural factors (stent diameter $<3 \mathrm{~mm}$, paclitaxeleluting stent and vein graft intervention) that reflect ischemic risks; while the only negative factor, age, reflects bleeding risks [6].

In the DAPT cohort, the median score was 2 with normal distribution [6]. Among these 11648 patients, ischemia (myocardial infarction or stent thrombosis) occurred in 348 (3.0\%) and bleeding (GUSTO moderate or severe bleeding) in $215(1.8 \%)$ during the study period. DAPT score had a C-index of 0.70 predicting ischemia and 0.68 predicting bleeding. As expected, there was opposite graded relationships (more ischemia but less bleeding events with higher score and vice versa). Continued thienopyridine versus placebo was associated with reduced ischemic events among the 5917 patients with score $\geq 2(2.7 \%$ vs. $5.7 \%$; $\mathrm{P}<0.001)$ but not among the 5731 patients with score $<2(1.7 \%$ vs. $2.3 \%$; $\mathrm{P}=0.07$; Interaction $\mathrm{P}$ value $<0.001)$. Continued thienopyridine was not associated with bleeding among the former group ( $1.8 \%$ vs. $1.4 \% ; \mathrm{P}=0.26)$ but was associated with bleeding among the latter group $(3.0 \%$ vs. $1.4 \%$; $\mathrm{P}<0.001$; Interaction $\mathrm{P}$ value $=0.02$ ). In the PROTECT trial validation cohort, the $\mathrm{C}$-index of the DAPT score was both 0.64 in predicting ischemia and in predicting bleeding [6].

\section{PRECISE-DAPT versus DAPT}

The strength of the DAPT score is that it predicts both ischemia and bleeding so that an overall assessment can be made to tailor antiplatelet therapy. The weakness is that it is based on a cohort of "uneventful" patients finishing a year of DAPT therapy so it is not relevant in the early post-PCI period. Also, the DAPT cohort included many patients receiving early generation DES (including about a quarter with paclitaxel eluting stents) and about a seventh with bare metal stent; and the C-index is relatively low in the external validation cohort.

Given that the DAPT cohort differs from the PRECISE-DAPT cohort in that patients had no bleeding in the first year on DAPT, it is no surprise that neither previous bleeding nor low haemoglobin (2 of the 5 PRECISE-DAPT factors) is present in the DAPT score. On the contrary, age is included in both scores and treated in somewhat similar manner-a 3-level parameter in DAPT ( 0 for age $<65,-1$ for age 65 to $<75,-2$ for age $>75$ ) and a continuous parameter from 50 to 90 years in PRECISE-DAPT.

Theoretically, one anti-platelet therapy strategy based on bleeding risks alone is only applicable to one pre-defined level of ischemic risk. PRECISE-DAPT score does not assess the underlying ischemic risks, so utility may be limited. As previously discussed in the journal [3], there was another recent patient-level meta-analysis [7] of 11473 patients with coronary stenting from 6 randomized trials $(58.5 \%$ with stable disease) which showed that the initial presentation altered outcome after different durations of DAPT: Patients with $\geq 6$ month
DAPT tended to have higher 1-year event rates of myocardial infarction or stent thrombosis than patients with 1-year DAPT if they had unstable disease (HR 1.48; 95\% CI, 0.98-2.22) but not if they had stable disease (HR 0.93; 95\% CI, 0.65-1.35).

In both DAPT score and PRECISE-DAPT score there is no evaluation of the degree of platelet suppression achieved. In another editorial in the journal [2], the ANTARCTIC trial was discussed where replacing prasugrel by clopidogrel was done in $\sim 40 \%$ of patients when platelet function test showed strong/excessive suppression 2 weeks after stenting. This "step-down" was apparently without any problems and therefore cost-saving.

In the very recent open-label, single center, randomized TOPIC trial [8], investigators followed 645 patients treated with PCI for acute coronary syndrome who had an uneventful course in the first month while taking aspirin and prasugrel/ticagrelor. Patients were then randomized to switched DAPT (to aspirin and clopidogrel) or unchanged DAPT (continuing original drug regimen). The composite primary endpoint (cardiovascular death, urgent revascularization, stroke and bleeding as defined by the Bleeding Academic Research Consortium classification $\geq 2$ ) at 1 year occurred less frequently in the switched DAPT group than in the unchanged DAPT group $(13.4 \%$ vs. $26.3 \%$; $\mathrm{P}<0.01)$, the difference being explained by bleeding $(4.0 \%$ vs. $14.9 \%, \mathrm{P}<0.01)$. Given that clopidogrel is a pro-drug requiring hepatic activation, its antiplatelet effect is dependent on the cytochrome P-450 2C19 allele status [1-3]. Preferably, the TOPIC trial findings need to be repeated with genetic and/or platelet function analyses.

\section{Conclusion}

In conclusion, tailoring DAPT after coronary stenting can be improved by scoring methods. This area of clinical science should be further explored given that coronary stenting is frequently performed and current second generation DES technology has matured. The data presented from PRECISE-DAPT and DAPT score studies are interesting. However, observational data are confounded by both measured and unmeasured parameters [9]. Large multicentre randomized clinical trial testing different antiplatelet regimes guided by a scientifically derived score incorporating ischemia and bleeding risk factors will improve management.

\section{References}

1. Wong CK (2012) Measuring platelet reactivity after clopidogrel-has it reached the end of the road. Cardiovasc Pharm 1: e105.

2. Wong CK (2016) From arctic to antarctic: is there any remaining role for platelet function testing to tailor therapy after coronary stent implantation. Cardiovasc Pharm 5: e132.

3. Wong CK (2017) Trials evaluating ticagrelor in cardiovascular disease: will it reign supreme in the anti-platelet world. Cardiovasc Pharm 6: el33.

4. Costa F, van Klaveren D, James S (2017) PRECISE-DAPT study investigators. Derivation and validation of the predicting bleeding complications in patients undergoing stent implantation and subsequent dual antiplatelet therapy (PRECISE-DAPT) score: a pooled analysis of individual-patient datasets from clinical trials. Lancet 389: 1025-1034.

5. Mauri L, Kereiakes DJ, Yeh RW (2014) DAPT Study Investigators. Twelve or 30 months of dual antiplatelet therapy after drug-eluting stents. N Engl J Med 371: 2155-2166.

6. Yeh RW, Secemsky EA, Kereiakes DJ (2016) DAPT study investigators. Development and validation of a prediction rule for benefit and harm of dual antiplatelet therapy beyond 1 year after percutaneous coronary intervention. JAMA 315: 1735-1749. 
Citation: Wong CK (2017) Tailoring Dual Antiplatelet Therapy after Coronary Stenting: The PRECISE-DAPT Score versus the DAPT Score. Cardiovasc Pharm Open Access 6: e135. doi:10.4172/2329-6607.1000e135

Page 3 of 3

7. Palmerini T, Della RD, Benedetto U (2017) Three, six, or twelve months of dual antiplatelet therapy after des implantation in patients with or without acute coronary syndromes: an individual patient data pairwise and network meta-analysis of six randomized trials and 11,473 patients. Eur Heart J 38 : 1034-1043.

8. Cuisset T, Deharo P, Quilici J (2017) Benefit of switching dual antiplatelet therapy after acute coronary syndrome: the TOPIC (timing of platelet inhibition after acute coronary syndrome) randomized study. Eur Heart J.
9. Wong CK (2015) Time for a reappraisal: How much has the last 10 years of "mainstream" STEMI research impacted on STEMI outcome. Cardiovasc Pharm 4: e129. 